\title{
De la red al piso de protección social: la trayectoria de la política social en los Organismos Multilaterales
}

\author{
Social Safety, from net to floor: the path of social policy through Multilateral Organizations
}

DOI: https://doi.org/10.22456/2178-8839.106693

Pablo Nemiña

Consejo Nacional de Investigaciones Científicas y Técnicas, Facultad Latinoamericana de Ciencias Sociales, Universidad Nacional de San Martín, Buenos Aires, Argentina pnemina@flacso.org.ar

Julián Echandi Universidad Nacional de San Martín, Buenos Aires, Argentina julianechandii@gmail.com

\begin{abstract}
Resumen
Desde mediados de la década de 1990 hasta la actualidad, el paradigma dominante de política social global viró desde la Red de Seguridad Social al Piso de Protección Social. El objetivo de este artículo es analizar el proceso político de transformación de la política social global promovida por los organismos multilaterales. En base a una metodología cualitativa, centrada en el análisis documental, se propone que cada paradigma de política social está vinculado a los postulados del modelo de desarrollo vigente. Mientras que en el marco del Consenso de Washington el Banco Mundial promueve la Red de Protección Social, entendida como políticas sociales focalizadas y excepcionales frente al optimismo en las soluciones de mercado; luego de la crisis financiera de 2008, e inspirado por la experiencia de las transferencias monetarias condicionadas en América Latina, la OIT impulsa el Piso de Protección Social, que comprende un enfoque de la política social más amplio, cuasi universal y basado en derechos. No obstante, los gemelos de Bretton Woods abrazaron con menos ímpetu este paradigma.
\end{abstract}

Palabras-claves: Política social; Post Consenso de Washington; América Latina.

\begin{abstract}
From the mid-1990s to the present, the dominant global social policy paradigm shifted from the Social Safety Net to the Social Protection Floor. The objective of this article is to analyse the political process of transformation of global social policy promoted by multilateral organizations. Based on a qualitative methodology, centred on documentary analysis, it is proposed that each paradigm of social policy is linked to the postulates of the current development model. While during the Washington Consensus wave the World Bank promoted the Social Safety Net, understood as focused and exceptional social policies in the face of optimism in market solutions; following the 2008 financial crisis, and inspired by the experience of conditional cash transfers in Latin America, the ILO promotes the Social Protection Floor, which includes a broader, quasi-universal and rights-based approach to social policy. However, the Bretton Woods twins embraced this paradigm less vigorously
\end{abstract}

Keywords: Social Policy; Post Washington Consensus; Latin America. 


\section{Introducción}

La crisis del Estado Keynesiano impulsó el progresivo deterioro de la condición salarial (CASTEL, 1997). El sistema de protecciones pensado para un mundo organizado en torno al contrato de trabajo por tiempo indeterminado, entró en tensión debido a las reformas socioeconómicas implementadas en los países en desarrollo bajo la inspiración del Consenso de Washington (CW). Este nuevo modelo de desarrollo que ponderó la prudencia macroeconómica, la orientación externa, la liberalización interna y las políticas de libre mercado (MARANGOS, 2008, p.227) quebró el mercado de trabajo y, por lo tanto, el sistema de protección social como se lo conocía hasta ese entonces. La heterogeneización de las formas de empleo sintetizadas en la figura del precariado aumentaron la incertidumbre e inseguridad, y contribuyeron a consolidar al desempleo como situación duradera para crecientes franjas de la población (STANDING, 2014). Sin embargo, durante la década de 1980 y buena parte de 1990 se subestimó la importancia de la política social, ya que se esperaba que el crecimiento derramara a través de los mecanismos de mercado hacia los sectores más vulnerables.

En los albores del nuevo milenio se dio la transición hacia el Post Consenso de Washington (PCW), un nuevo paradigma de desarrollo que tenía como preocupación central enfrentar la pérdida de cohesión social y avanzar en la construcción de un nuevo contrato social. Si bien ratificaba la centralidad de las políticas de liberalización, apertura y prudencia fiscal y monetaria alentadas por el CW, incorporó la preocupación por la dimensión social y el contexto político institucional en el que se llevaban adelante las reformas (GÜVEN, 2018).

En línea con el cambio en el paradigma de desarrollo, durante esta etapa los organismos multilaterales emprendieron distintas iniciativas con el fin de promover una agenda social que revirtiera la creciente deslegitimación de la globalización (DEACON, 2013a). La literatura de Relaciones Internacionales reconoce que las instituciones internacionales promueven agendas particulares en su interacción con los Estados, pero ¿de dónde surgen y cómo se despliegan? Mientras que para los enfoques racionalistas el cambio refleja la adaptación al entorno material y normativo, los constructivistas enfatizan la capacidad de las instituciones internacionales -en tanto actores sociales-de crear y establecer agendas. En este trabajo proponemos que la incorporación y el impulso de la agenda social en los organismos multilaterales es el resultado de una combinación de adaptación al contexto y el impulso de la agenda por parte de funcionarios y otros actores clave (VETTERLEIN, 2015). Asimismo, se trató de un proceso no lineal, en el cual se observaron disputas políticas entre los organismos y dentro de sus estructuras, como así también fuertes discusiones en torno al paradigma en el cual se iban a enmarcar las políticas sociales.

Planteamos como objetivo analizar los cambios ocurridos en el paradigma de política social alentado por los principales organismos multilaterales involucrados en el proceso de cooperación global en temas sociales durante los últimos treinta años. Para esto, apoyados en una metodología cualitativa basada esencialmente en el análisis bibliográfico y documental, analizaremos el proceso de diseño de la política social que los organismos desarrollaron entre el Consenso y el Post Consenso de Washington, centrándonos en el proceso de diseño e implementación de la Red de Seguridad Social y la transición hacia el Piso de Protección Social luego de la crisis financiera de 2008. Planteamos como contribución primordial mostrar que el proceso de incorporación y cambio de la política social en los organismos internacionales fue gradual y para nada homogéneo. Con relación al gradualismo, éste refleja la combinación de la adaptación al cambio de paradigma de desarrollo, la ponderación experiencias regionales exitosas -como los programas de transferencia monetaria condicionada- y el liderazgo por parte de actores específicos, como la OIT. Respecto de la heterogeneidad del cambio, éste remite a las diferentes agendas y márgenes de acción que posee cada organismo. Por ejemplo, la relegitimación del FMI luego de la crisis financiera de 2008 junto a su mandato restringido en lo que a temas sociales se refiere, contribuye a entender la distancia de este organismo con la agenda inicialmente dominante.

El trabajo se divide en cinco partes. En la primera repasamos los principales antecedentes sobre políticas sociales y organismos internacionales. En la segunda, analizamos el surgimiento de Red de Seguridad Social como principio rector de la política social y su vinculación con el paradigma del Consenso de Washington. En la tercera sección caracterizamos 
el Post Consenso de Washington junto a los cambios y continuidades que posee con el CW. Luego, estudiamos el surgimiento y desarrollo de las transferencias monetarias condicionadas en América Latina y su impacto en el paradigma de Piso de Seguridad Social, cuyo surgimiento y conflictivo desarrollo analizamos en la quinta sección. Cierra el trabajo un apartado de conclusiones.

\section{Política Social y Organismos Internacionales}

La política social se caracteriza por ser un concepto polisémico, heterogéneo y múltiple. Entendiendo que no puede ser reducido a una definición unívoca, con un objetivo analítico identificamos dos grandes grupos que han abordado su estudio. Por un lado, están aquellos que lo hicieron entendiendo la política social como un mecanismo político institucional a través del cual el Estado interviene de diversas maneras, con el fin de garantizar la reproducción de la fuerza de trabajo (CENA, 2014). Siguiendo este planteo, consideramos que las políticas sociales son instituciones centrales en los procesos de desmercantilización de los derechos sociales (ESPING-ANDERSEN, 1993, 2000) ya que ordenan recursos y responsabilidades ligadas al acceso a las distintas dimensiones de lo que se defina como bienestar: ingresos, empleo, educación, vivienda y salud, entre otras. Otros autores han analizado las políticas sociales centrándose en su vínculo bidireccional con la estructura socio-económica (ADELANTADO et al, 1998; FALEIROS, 2000). En este sentido, este tipo de intervenciones no sólo funcionan como compensaciones de las desigualdades sociales producidas por el mercado, sino que también cumplen la tarea de definirlas y configurarlas.

Específicamente en lo que respecta al rol de la política social para los organismos multilaterales, algunos autores han orientado su análisis a las políticas propuestas por la Comisión Económica para América Latina y el Caribe (CEPAL), la Organización para la Cooperación y el Desarrollo Económicos (OCDE) y OXFAM, con el objetivo de enfrentar el aumento de la desigualdad del ingreso y de la pobreza (BARBA SOLANO, SILVA MEDINA, 2018); otros reconstruyeron la relación entre el Banco Interamericano de Desarrollo y el Banco Mundial, en el diseño, implementación y evaluación de las políticas sociales desarrolladas en los países de América Latina (MALLARDI et al, 2015). También se ha evaluado el impacto de la implementación de las políticas de Transferencias Monetarias Condicionadas (TMC) (FISZBEIN, SCHADY, 2009; LAVINAS, 2013; VALENCIA LOMELÍ, 2008) y las tensiones entre el paradigma universalismo-focalización de la política social a lo largo de los últimos veinticinco años (BARBA SOLANO, 2018; FRANCO, 1996).

A pesar de que los Estados nacionales son los encargados de implementar las políticas sociales en sus respectivos territorios, los organismos internacionales logran incidir sobre el diseño de las mismas (BABB, CHOREV, 2016). Instituciones económicas como el FMI y el Banco Mundial lo hacen directamente a través de condicionalidades incluidas en los préstamos y de recomendaciones brindadas en el marco de asesoramientos técnicos en materia social (PARK, VETTERLEIN, 2010). A partir de la definición de Bob DEACON (2007), entendemos a la política social global como las prescripciones de política social de los actores globales dirigidas a los gobiernos nacionales y las políticas sociales propiamente supranacionales que pueden incluir mecanismos de redistribución, regulación y promoción de derechos sociales a escala global.

\section{La red de seguridad social. Políticas transitorias y focalizadas}

Hacia finales de la década de 1980 las Instituciones Financieras Internacionales tuvieron un rol destacado como impulsoras de la transición al capitalismo en los países de Europa del Este y las reformas de mercado en los países en desarrollo. A través de las condicionalidades que acompañaban a sus programas de financiamiento y de la asistencia técnica y recomendaciones basadas en su legitimidad como autoridad experta, el FMI y el Banco Mundial promovieron políticas inspiradas en el Consenso de Washington (WOODS, 2007). Nos referimos a un conjunto de reformas político- 
económicas impulsadas ante el fin de la Guerra Fría y la caída de los regímenes socialistas, que definieron de manera significativa el paradigma de desarrollo durante la década del noventa. Las mismas fueron presentadas como políticas consensuadas y consideradas necesarias por las instituciones de Washington (el FMI, el Banco Mundial y el Tesoro de los Estados Unidos) para América Latina, que atravesaban años de estancamiento económico debido a la crisis de la deuda externa (SERRA et al, 2008).

Los impulsores del Consenso de Washington destacaban como una de las principales causas del desajuste macroeconómico de la región a los déficits de las cuentas públicas, por tal motivo, su primera recomendación apuntó a la disciplina fiscal. Esto se relacionaba con la focalización del gasto público, es decir, el redireccionamiento de los subsidios a la cobertura básica de Educación y Salud, y de las inversiones estatales hacia el sector de infraestructura pública, que fue el segundo punto señalado en el decálogo. Por otra parte alentaban una reforma impositiva que ampliara la base tributaria; la liberalización de las tasas de interés y el sostenimiento de tipos de cambio competitivos, que incrementaran las exportaciones. En términos comerciales, promovían la liberalización del comercio a través de la eliminación de barreras no arancelarias y de impuestos a las exportaciones; además, se sumó la apertura a las Inversiones Extranjeras Directas, la privatización de empresas públicas y la desregulación de los mercados financieros, productivos y laborales, como formas de impulsar un mayor nivel de competencia y de estímulo al crecimiento (MORANDÉ, 2016). Esta nueva estrategia de desarrollo se basaba en dos pilares fundamentales: la estabilidad económica mediante el ajuste fiscal y la ortodoxia del mercado, y en la drástica reducción del tamaño del Estado y su rol en la economía (SERRA et al, 2008).

Si bien impulsaron el crecimiento y la modernización económica, las reformas de mercado generaron un aumento de la desigualdad, la pobreza y el desempleo en los países donde fueron implementadas con mayor énfasis (KESSLER, BENZA, 2020), lo cual alentó las voces críticas tanto de los movimientos sociales como de algunos organismos internacionales. A las tempranas advertencias de UNICEF (CORNIA et al, 1987) que promovió el "ajuste con rostro humano", se sumó la OIT, que criticaba el costo social del ajuste y su impacto negativo sobre el empleo. Si bien ambas instituciones aceptaban como inevitables las reformas, promovían la inclusión de políticas sociales compensatorias en los programas de financiamiento para atemperar el impacto sobre la población más vulnerable a los shocks económicos. Esto se cristalizó en el concepto de Red de Seguridad Social (en adelante RSS), que desde mediados de la década de 1990 progresivamente cobró importancia en el discurso social de las IFIs globales (TOWNSEND, 2004; DEACON, 2007). El concepto remite a la imagen circense de una red que amortigua la caída de un acróbata ante el caso excepcional de que el acto salga mal, previniendo daños severos sobre el artista. De mismo modo, la RSS sería un mecanismo que reduciría los daños sobre la población más vulnerable en situaciones excepcionales.

Si bien inicialmente las IFIs mostraron un optimismo exagerado en la autorregulación del mercado y subestimaban la importancia de contemplar políticas sociales específicas (MAUL, 2019), como respuesta a las críticas que denunciaban el impacto social de los programas de ajuste el Banco Mundial promovió la Red de Seguridad Social. La RSS era un concepto "paraguas" que abarcaba un amplio abanico de políticas sociales como seguros de desempleo, programas de trabajo público, subsidios en alimentos, vivienda y servicios, entre otras, que compartían dos características: se trataba de políticas son políticas sociales focalizadas específicamente en la población más pobre con el objetivo de alivianar las consecuencias sociales negativas de una estrategia macroeconómica de ajuste, y a su vez son de carácter excepcional dado que sólo se justifican cuando se ve amenazada la subsistencia de una parte de la población. Así, la focalización y la excepcionalidad fueron dos de los principios rectores del concepto de RSS desde las primeras acepciones del Banco Mundial. (DEACON, 2013a).

El FMI tuvo una trayectoria similar a la del Banco. Hasta principios de los '90 el organismo sobreestimó el impacto positivo de las reformas de mercado y delegó a los países deudores la administración de las consecuencias sociales de las políticas de desregulación, con el argumento de que los temas sociales excedían sus áreas de responsabilidad (ABRAMS, 2017). Ante el progresivo deterioro de los indicadores sociales, y presionado por el Banco Mundial y otras organizaciones 
de la ONU (VETTERLEIN, 2015), incorporó en 1993 la RSS en el diseño de sus programas de financiamiento. El FMI definió la RSS como políticas sociales ad hoc o permanentes que tenían como objetivo mitigar los efectos adversos de reformas económicas sobre la población de bajos ingresos, entre los cuales se destacaban subsidios limitados para la satisfacción de las necesidades básicas (esencialmente alimentos), arreglos de seguridad social (como pensiones y beneficios en caso de desempleo) y posibles programas de empleo público (IMF, 1993, p.23).

Uno de los argumentos que sustentaron la incorporación de medidas sociales a los programas del FMI era contribuir a garantizar la sustentabilidad política de las medidas económicas. Dicho de otro modo, financiar políticas sociales que mitigaran los efectos de los ajustes estructurales ayudaría a evitar que la presión social volviera políticamente inviable la continuidad de las reformas. Sin embargo, este proceso no estuvo exento de controversias en el Directorio del organismo. Mientras que algunos directores argumentaban que el FMI no tenía la expertise necesaria para diseñar políticas sociales, otros reconocían la relevancia de las medidas, pero resaltaban la necesidad de afianzar la cooperación con el Banco Mundial, en tanto organismo experto en esa área (ABRAMS, 2017).

Ante la profundización de las desigualdades, las políticas sociales fueron ganando espacio en el discurso y las prácticas de las Instituciones Financieras Internacionales en la periferia. Sin embargo, pronto enfrentaron un dilema. Si las reformas neoliberales impulsaron cambios en la estructura social que cristalizaban una distribución más desigual del ingreso, la viabilidad política de estas transformaciones requería respuestas sociales permanentes y más abarcadoras antes que focalizadas y transitorias. El Estado debía asumir la tarea para la cual mercado se había mostrado incapaz. Esto será el puntapié para el diseño del Post Consenso de Washington, un reformulación del CW que contemplará mayor espacio para la intervención pública, aunque sin resignar los lineamientos centrales de las reformas.

\section{Un nuevo paradigma: el Post Consenso de Washington}

Las sucesivas crisis financieras acontecidas durante la segunda mitad de la década de 1990 generaron un creciente malestar en la sociedad civil hacia las políticas pro mercado inspiradas en el Consenso de Washington y las instituciones financieras globales que las promovieron (WOODS, 2007). En este sentido la crisis en el Sudeste Asiático significó un parteaguas: la región que había sido ponderada por el FMI y el Banco Mundial como modelo, se veía inmersa en una profunda crisis económica ante la cual las medidas recomendadas por los organismos no hacían más que agravar el impacto social y económico de la crisis (ÖNIS, ŞENSES, 2005).

Además del creciente descontento de la ciudadanía, el FMI afrontó las críticas hasta del propio Banco Mundial. En su rol de economista jefe del Banco, a finales de los años noventa Stiglitz apuntó contra el liberalismo extremo y acuñó el término Post- Consenso Washington en la Conferencia WIDER (STIGLITZ, 1998); por otra parte, el Banco publicó "La Larga Marcha. Una agenda de reformas para la próxima década en América Latina y el Caribe”, un informe en el que, de alguna manera, reconocía las limitaciones del CW.

En este contexto emergió el Post-Consenso de Washington (en adelante, PCW), una revisión del consenso original (CW) que complementó la orientación aperturista y pro mercado de las políticas económicas con un enfoque novedoso en la dimensión sociopolítica del desarrollo (GÜVEN, 2018), ampliando y profundizando la agenda neoliberal (ÖNIS, ŞENSES, 2005) sin abandonar los fundamentos metodológicos e ideológicos de la teoría neoclásica (LESAY, 2012). El PCW parte de dos supuestos, interrelacionados entre sí. Primero, el problema del CW residía en que presionó por un "excesivo” achicamiento del Estado, al tiempo que centró su atención sólo en la dimensión cuantitativa: había que reducirlo de tamaño. Segundo, las fallas del CW mostraban que era necesario "ayudar" al mercado para que funcionara correctamente. Los resultados dispares que mostraban las reformas explicitaban que el mercado no era capaz de promover competitividad por sí solo. Eran variados los ejemplos en los cuales se había reemplazado una asignación ineficiente de recursos como 
consecuencia de la intervención del Estado, por otra similar como resultado del libre mercado (un caso testigo lo constituía el sector financiero).

Las reformas propuestas por el PCW diferían del CW en el alcance de su objetivo, los instrumentos y la autoría de las mismas. Mientras que el CW planteó un objetivo limitado (sostener el crecimiento económico), una pequeña serie de instrumentos de política (centrados en política económica macro) y autoría externa (de Washington se exportaba hacia las regiones emergentes); el PCW proponía un objetivo más amplio y complejo (consolidar el desarrollo), más y más amplios instrumentos y metas (centrados en cuestiones tanto macro como micro, que muchas veces excedían lo estrictamente económico) y la posibilidad de adecuar las políticas a la coyuntura de cada país (STIGLITZ, 1998).

Ante esta situación, el PCW planteó la necesidad de implementar una serie de reformas de segunda generación centradas en reposicionar y reforzar al Estado. En especial, se destaca aumentar la regulación de la economía, a fin de evitar fallas de mercado como los monopolios y fomentar la libre competencia; fortalecer al Estado mediante el refuerzo de sus capacidades financiera y administrativa; aumentar la inversión estatal en educación y tecnología; e impulsar la implementación de políticas sociales. Con relación a este último punto, si bien el Consenso de Washington no vetaba el uso de políticas sociales, el énfasis en la consolidación fiscal llevó usualmente a recortes en los gastos sociales (BIRDSALL, FUKUYAMA, 2011).

El PCW planteaba aumentar la intervención del Estado y reforzar sus capacidades. Se trataba de lograr un Estado con mayor intervención y mejores capacidades, que reemplazara al Estado pequeño y débil que había resultado del CW. Paralelamente, buscaba superar la antinomia Estado / mercado. Sugería que así como los límites de la industrialización por sustitución de importaciones habían demostrado la incapacidad del Estado de garantizar por sí mismo el desarrollo, las fallas de las reformas neoliberales evidenciaban las limitaciones del mercado. Esto mostraba la necesidad de integrar al mercado con el Estado, en tanto el primero no conseguía funcionar naturalmente de manera óptima. Por ello, menos que considerarse opuestos, cada uno podía tomar elementos del otro a fin de lograr una complementación entre ambos. Así pueden entenderse propuestas como hacer eficiente al Estado (a partir de la reforma basada en la nueva administración pública, que planteaba superar la organización burocrática por la gerencial) o regular al mercado para fomentar la competencia (por ejemplo, mediante la sanción de leyes anti-monopolio).

Otros aspectos novedosos en las propuestas planteadas en el PCW son la capacidad de 'Ownership' que se le concede a cada país en el diseño de las políticas y el incentivo a la participación de las bases sociales. Estas dos iniciativas se presentan principalmente como respuestas a la erosionada legitimidad política que mostraban los gobiernos, tanto frente a la sociedad civil como también a los prestamistas, a la hora de emprender reformas y programas de gobierno durante el CW. Esto estimuló la creación de consensos para llevar adelante cambios significativos, lo cual alentó la participación de la comunidad en el diseño de políticas y permitió aportar adecuar a las necesidades locales los generales de política, tomando en consideración las experiencias históricas nacionales, el rol de los actores e intereses en juego (ZURBRIGGEN, 2011) y entendiendo que los comportamientos sociales no se modifican simplemente mediante imposiciones externas (ÖNIS, ŞENSES, 2005; STIGLITZ, 1998; STIGLITZ, 2008).

Si bien el paradigma de la ownership favoreció la consideración del contexto local, no está exento de limitaciones. Según WILLIAMS (2006, p.10), refiere a "un discurso y un conjunto de prácticas dirigidas a superar ciertos problemas asociados con la fragmentación del control y la autoridad sobre el desarrollo" que remiten a la construcción de una nueva agenda de gobernanza global. Si las revueltas populares de finales de la década de 1990 eran un freno a la globalización neoliberal, la ownership aparecía como el margen de personalización doméstica tolerable por los organismos internacionales para hacer viable políticamente la implementación de los programas de reforma. En el mismo sentido, convertir a los países prestatarios en socios les permitía compartir los costos políticos ante la implementación de reformas impopulares, en una clara estrategia de supervivencia institucional. 


\section{América Latina como pivote del Piso de Protección Social}

La limitada red de seguridad social implementada durante la década de 1990 se mostró incapaz de dar respuesta a los principales problemas que presentaba la estructura socioeconómica latinoamericana: la creciente desigualdad y la amplia franja de trabajadores informales. Según RIGGIROZZI (2020, p.5), durante la década de 1990, seis de cada diez nuevos empleos creados en la región fueron informales; mientras que a finales de esa década, 211 millones de latinoamericanos vivían bajo la línea de pobreza (un 55\% más que dos décadas atrás).

Ante la incapacidad de las tímidas medidas sociales focalizadas enmarcadas en el paradigma de la RSS en morigerar el deterioro de las condiciones de vida de la población, México y Brasil impulsaron los programas Progresa/Oportunidades y Bolsa Escola en 1997 y 2001 respectivamente. Se trataba de programas asistencia social que en lugar de distribuir recursos a través de subsidios o la entrega de alimentos, otorgaban una transferencia monetaria condicionada (TMC) a familias de bajos ingresos. Estos programas implicaban una transferencia monetaria mensual a cambio de (y como incentivo para) que las familias garantizaran la asistencia escolar y los chequeos médicos de sus integrantes. Los programas de TMC permitieron combinar la focalización característica del enfoque neoliberal con el objetivo - afín a la agenda ampliada del PCW - de reforzar el capital humano sin afectar la lógica del mercado a través de la “distorsión” de los precios relativos (RIGGIROZZI, 2020, p.6).

A partir de las iniciativas pioneras de México y Brasil, los programas de TMC cobraron un impulso significativo en la región, los cuales en 2010 alcanzaron al 22,7\% de la población, del 3,6\% en el 2000 (Ibid, p.8). Otros ejemplos destacados de programas TMC han sido Bolsa Familia (2004) Brasil, la Asignación Universal por Hijo (2009) en Argentina y el Plan AUGE (Plan de Acceso Universal de Garantías Explícitas) (2005-2009) luego GES (Garantías Explícitas en Salud) en Chile. Con el impulso de estas medidas, la región logró reducir la tasa de pobreza al 28,3\% al 2012, del 43,8\% en 1999, y más de un tercio el nivel de indigencia, del 18,6\% hasta el 11,3\%, durante el mismo período (OIT, 2014).

De la mano de los gobiernos de la "ola rosa" progresista y la bonanza derivada del boom de las materias primas, los programas de TMC fueron el pivote de la política social posneoliberal, la cual se caracteriza por nuevas políticas y discursos que expanden derechos y atienden necesidades sociales recientemente reconocidas, en particular, las identidades (RIGGIROZZI, 2020; RIGGIROZZI, TUSSIE, 2017). Si bien lograron desplegar reducir la pobreza y la indigencia, generaron una inclusión a través de mecanismos de mercado que no lograron superar las desigualdades estructurales, debido -en parte- a su fondeo esencialmente pro cíclico (LAVINAS, 2013). Al respecto, la OIT (2014) señaló que al 2012 el 23,2\% de los residentes en zonas urbanas y el 48,6\% de los habitantes de zonas rurales se encontraban aún bajo la línea de pobreza, e inclusive un tercio de los trabajadores urbanos, en su mayoría mujeres, permanecía directamente fuera del paraguas de los sistemas formales de protección social.

La experiencia de los programas de TMC en América Latina sirvió como inspiración al Piso de Protección Social (en adelante, PPS), un modelo de política social pensado para garantizar un piso mínimo de bienes y servicios que no fuera inferior a la línea de pobreza, los cuales incluyen un conjunto de derechos básicos como el acceso a la salud, la nutrición y la educación y transferencias de ingresos (CICHON et al, 2011). Se trata de un enfoque de política social adaptable a las necesidades, prioridades y recursos nacionales que complementa la seguridad social (OIT, 2011, p. XXVIII), y por tal motivo, es coherente con el paradigma del PCW en tanto respeta y alienta la ownership.

\section{La disputa em torno al piso de protección social}

Además del interés por expandir la experiencia latinoamericana, las reflexiones sobre las críticas de los movimientos sociales a las reformas de mercado y el impacto de la crisis financiera global de 2008 tuvieron un papel significativo para impulsar la iniciativa del Piso de Protección Social por parte de la OIT. 
Uno de los antecedentes más tempranos se encuentra en el informe de la Comisión Mundial sobre la Dimensión Social de la Globalización (OIT, 2004), donde se definía el concepto de Piso Social Global como una base socioeconómica que aspiraba a reconstruir la legitimidad menoscabada de la globalización y morigerar sus efectos sobre la desigualdad e inseguridad humanas. El planteo formó parte de la Agenda de Trabajo Decente de la OIT en 2006, y cobró centralidad al año siguiente con la llegada del nuevo Jefe del Departamento de Seguridad Social, Michael Cichon, quien promovió la Campaña por una Coalición para un Piso Social Global.

Pero es a partir de la crisis financiera de 2008 que la iniciativa del PPS cobrará preeminencia. La OIT incidió de manera relevante en la elaboración del documento "La crisis financiera mundial y su impacto en el trabajo del Sistema de las Naciones Unidas”, realizado por la Junta de coordinación de los Jefes Ejecutivos del Sistema de las Naciones Unidas en 2009. Allí se instaba a la concertación del sistema en ocho campos de políticas; particularmente en el campo de los Servicios sociales, empoderamiento y protección de las personas, aparecía la referencia a un Piso de Protección Social Global con el que se proponía garantizar "el acceso a servicio sociales básicos, vivienda, y el empoderamiento y la protección de los pobres y vulnerables" (JJE, 2009). Como importante espaldarazo a la iniciativa PPS, la OIT lograba el apoyo de la Organización Mundial de la Salud, UNICEF y el Departamento de Asuntos Económicos y Sociales de Naciones Unidas (UNDESA) (DEACON, 2013b).

Del proceso también participaron otros actores, como instituciones financieras multilaterales, representantes de países y miembros de ONGs, concretando una primera aproximación al PPS y delineando los costos y el espacio fiscal para su implementación. También fijaron compromisos para el monitoreo y evaluación de su aplicación, y el establecimiento de una red mundial de asesoramiento técnico y una plataforma de intercambio de conocimientos bajo la dirección de la OIT, que se constituyó como la Extensión Global de la Seguridad Social (GESS) (DEACON, 2013b). El objetivo de cooperación se tornó más evidente al establecer un Grupo Asesor de Protección Social a cargo de Michelle Bachelet, entonces Secretaria Adjunta de las Naciones Unidas, que tenía bajo su mandato la formulación, promoción y asesoramiento sobre la sistematización del concepto de PPS.

El PPS consolida su protagonismo en la agenda internacional entre 2010 y 2011 al lograr el respaldo de la Cumbre de los Objetivos de Desarrollo del Milenio, la 100a Sesión de la Conferencia Internacional del Trabajo, la Declaración de Brasilia firmada por países miembros latinoamericanos bajo el auspicio de la CEPAL, y la Cumbre de Jefes de Estado y Gobierno del G20 celebrada en Cannes. Los pronunciamientos llamaron a la coordinación entre las organizaciones internacionales, sobre todo entre la OIT y el FMI, para asesorar a los gobiernos nacionales en el diseño de las políticas fiscales necesarias para desarrollar el PPS. Durante la Conferencia Conjunta de 2010 en Oslo entre la OIT y el FMI, estos organismos se comprometieron a trabajar conjuntamente para explorar el PPS, dirigido a personas que se encontraban en la pobreza o en situaciones vulnerables dentro de un marco de políticas macroeconómicas sostenibles a mediano y largo plazo (ILO; IMF, 2012). Sin embargo los compromisos discursivos no permearon significativamente en el FMI y los avances en el campo de la política social fueron marginales. Alentado por la recuperación económica que siguió a la crisis financiera, el Fondo se enfocó en las políticas fiscales, monetarias, cambiarias y financieras (sus temas de expertise tradicional), y enfatizó progresivamente la consolidación fiscal (NEMIÑA; LARRALDE, 2020).

El FMI reconoció discursivamente la importancia del gasto social para alcanzar un nuevo contrato social post 2008 (MERLING, 2019), pero sus recomendaciones no mostraron cambios significativos. Si bien diversos préstamos otorgados a países de bajos ingresos luego de la crisis financiera incluyeron un compromiso de piso de gasto social (que el Fondo denomina 'Social Spending'), en la práctica se trató de una categoría residual en la cual se agrupó una amplia gama de gastos sociales, sin ningún tipo de delimitación de montos ni especificidades de las políticas sociales que se llevan adelante en cada país miembro. Tampoco los desembolsos corrían el riesgo de ser suspendidos si no se cumplía con lo estipulado en este punto, ya que en la mayoría de los casos los compromisos de gasto social eran metas indicativas, cuyo incumplimiento no amerita sanciones formales (KENTIKELENIS; STUBBS; KING, 2016). Las críticas señalaron que el Fondo 
pareció más interesado en "llenar un formulario" (GCSPF, 2019, p.9) que en alinearse de manera comprometida con la OIT y UNICEF, a fin de adaptarse a los estándares de la comunidad internacional en la agenda social (IEO, 2017; GCSPF, 2019; MERLING, 2019).

Mientras que el FMI desarrolló su propia agenda social y mostró indiferencia ante la iniciativa PPS, el Banco Mundial disputó abiertamente a la OIT el liderazgo entre los organismos multilaterales en el diseño de la política social. Se trata de una trayectoria comprensible, dada la centralidad de los temas sociales en el mandato del Banco (VETTERLEIN, 2007). En este sentido, durante la Cumbre de Los Cabos del G20 (2012) el Banco presentó una contrapropuesta para la articulación de la cooperación global en políticas sociales, que implicaba la creación de la Junta Interagencial de Protección Social, compuesta por distintas organizaciones internacionales aunque bajo dirección del Banco (G20-DWG, 2012). El apoyo a la Junta por parte de Vinicius Pinheiro, uno de los armadores políticos internos de la OIT, fue clave para diluir la iniciativa PPS, que desde entonces fue desplazada por la JCIPS (DEACON, 2014). A pesar de haber sostenido que los PPS formaban parte de sistemas integrales de protección social, la JCIPS los excluyó progresivamente de sus deliberaciones.

\section{Reflexiones finales}

Así como la transición al modelo de desarrollo del Post Consenso de Washington no implicó un quiebre radical con respecto al Consenso de Washington, la política social desarrollada en los últimos treinta años por los organismos multilaterales muestra aspectos novedosos y continuidades. El cambio en la política social promovida por las organizaciones internacionales, que acompañó la transformación del modelo de desarrollo global imperante, fue progresivo y convivió con tensiones de diverso calibre. Se pasó de una concepción restringida, focalizada y excepcional como fue el concepto de Red de Protección Social, que tenía como central destinatarios a la población más vulnerable, a una formulación más amplia, duradera y cuasi universal en su diseño e implementación como fue el Piso de Protección Social, impulsado con ímpetu desde los organismos a partir de la crisis financiera global de 2008. En este proceso la experiencia de los PTC en América Latina constituyó un antecedente clave que sirvió de inspiración a un paradigma de políticas sociales centrado en la ampliación y consolidación de derechos.

Sin embargo, los organismos internacionales no aplicaron en sus propuestas el paradigma del Post Consenso de Washington ni ampliaron el abanico de políticas sociales de la misma forma. Los gemelos Bretton Woods fueron más reticentes a la aplicación de políticas de corte universalista e hicieron poco para avanzar en ese sentido. La propuesta de la OIT explicitó las resistencias por ampliar el marco normativo y de alcance de las políticas sociales con el fin de trascender la mirada asistencialista. Pese a los avances que se registraron en la transición de los modelos de desarrollo, el paradigma que enmarca el diseño de las políticas sociales continúa tensionado entre la focalización y la universalidad restringida, y no ha logrado trascender hacia un sistema universal e integrado de protección social.

Si bien luego de la crisis financiera de 2008 se observa una coincidencia entre las organizaciones internacionales en torno a garantizar mayor centralidad a la protección social en el diseño de políticas públicas, las disputas entre las instituciones sobre la imposición de los conceptos sobre los que se basa el diseño e implementación de las políticas sociales concretas limitan, hasta el momento, alcanzar cambios estructurales.

\section{Referencias}

ABRAMS, Alisa. The IMF's Role in Social Protection: Fund Policy and Guidance. IEO Background Document No. BD/17-01/01. Washington: IEO, 2017.

ADELANTADO, José et al. Las relaciones entre estructura y política sociales: una propuesta teórica. Revista Mexicana de Sociología, v.60, n. 3, p.123, 1998. 
BABB, Sarah; CHOREV, Nitsan. International organizations: Loose and tight coupling in the development regime. Studies in Comparative International Development, v.51, n.1, p.81-102, 2016.

BARBA SOLANO, Carlos; SILVA MEDINA, Yasodhara. El ADN de los informes internacionales sobre desigualdad realizados por la OCDE, la CEPAL y OXFAM durante la segunda década del siglo XX. En Midaglia, C.; Ordoñez, G.; Valencia, E. (coords.). Políticas sociales en América Latina en los inicios del siglo XXI: innovaciones, inercias y retrocesos, Buenos Aires: CLACSO, p.271-298, 2018.

BARBA SOLANO, Carlos. Liberalismo y universalismo en tensión. En Midaglia, C.; Ordoñez, G.; Valencia. E. (coords.). Políticas sociales en América Latina en los inicios del siglo XXI: innovaciones, inercias y retrocesos. Buenos Aires: CLACSO, p.75-108, 2018.

BIRDSALL, Nancy; FUKUYAMA, Francis. The Post-Washington Consensus: development after the crisis. Foreign Affairs, v.90, p.45-53, 2011 .

BUIRA, Ariel (Ed.). The IMF and the World Bank at sixty. London: Anthem Press, 2005.

CASTEL, Robert. La metamorfosis de la cuestión social. Crónica del salariado. Buenos Aires: Paidós, 1997.

CENA, Rebeca. Programas de transferencias condicionadas de ingresos y programas de empleo en Argentina: entre la responsabilización de los destinatarios y la individualización de la cuestión social. Boletín Científico Sapiens Research, v.4, n.1, p.38,2014

CICHON, Michael; BEHRENDT, Christina; WODSAK, Veronika. La iniciativa del Piso de Protección Social de las Naciones Unidas. Análisis de Políticas Internacionales. Friedrich-Ebert-Stiftung, Berlín, 2011.

CORNIA, Giovanni et al. Adjustment with a human face: protecting the vulnerable and promoting growth. Oxford: Clarendon Press, 1987.

DEACON, Bob. Career, carisma, conviction and the challenges facing the Social Protection Floor. Global Social Policy, v.14, n.3, p.406416,2014

DEACON, Bob. Global social policy and governance. UK: SAGE Publications. 2007.

DEACON, Bob. Global social policy in the making: The foundations of the social protection floor. Bristol: Policy Press, 2013a.

DEACON, Bob. The social protection floor and global social governance: Towards policy synergy. International Social Security Review, v.66, n.3-4, p.45-67, 2013 b.

ESPING-ANDERSEN, Gosta. Fundamentos sociales de las economías postindustriales. Barcelona: Ariel, 2000.

ESPING-ANDERSEN, Gosta. Los tres mundos del Estado de Bienestar. Madrid: Ediciones Alfons el Magnanim, 1993.

FALEIROS, Vicente. Las Funciones de la Política Social en el Capitalismo. En BORGIANNI, Elisabete; MONTAÑO, Carlos. La política social hoy. Madrid: Cortez editora. p.43-70, 2000.

FISZBEIN, Ariel ; SCHADY, Norbert. Transferencias Monetarias Condicionadas. Reduciendo la pobreza actual y futura. Washington: Banco Mundial, 2009.

FRANCO, Rolando. Los paradigmas de la política social en América Latina. Revista de la CEPAL, v.58, p.9-22, 1996.

GLOBAL COALITION FOR SOCIAL PROTECTION FLOOR [GCSPF]. The IMF's new policy framework on social protection. January 2019, recuperado de (19/8/20): http://www.socialprotectionfloorscoalition.org/2019/01/the-imfs-new-policy-framework-on-socialprotection/

GÜVEN, Ali. Whither the post-Washington Consensus? International financial institutions and development policy before and after the crisis. Review of International Political Economy, v.25, n.3, p.392-417, 2018.

IEO. The IMF and social protection. Evaluation Report. Washington: IEO, 2017.

ILO; IMF. Towards effective and fiscally sustainable social protection floors. PreliminaryDraft. Geneva: ILO, Washington: IMF, 2012. Recuperado de (19/8/20): https://www.ilo.org/wcmsp5/groups/public/---ed_protect/-.. soc_sec/documents/publication/wcms_secsoc_30810.pdf

IMF. Social Safety Net in Economic Reform, Internal Document EBS/93/34. IMF: Washington, DC, 1993.

KENTIKELENIS, Alexander; STUBBS, Thomas; KING, Lawrence. IMF conditionality and development policy space, 1985-2014. Review of International Political Economy, v.23, n.4, p.543-582, 2016.

KESSLER, Gabriel; BENZA, Gabriela. La ¿nueva? Estructura social de América Latina. Cambios y persistencias después de la ola de gobiernos progresistas. Buenos Aires: Siglo XXI Editores, 2020. 
LAVINAS, Lena. Latin America. Anti-Poverty Schemes Instead of Social Protection. Working Paper Series. Working Paper, n.51, 2013. Desigualdades.net, disponible en (19/8/20): http://www.nopoor.eu/publication/latin-america-anti-poverty-schemes-instead-socialprotection

LESAY, Ivan. How 'post' is the Post-Washington Consensus?. Journal of Third World Studies, v.29, n.2, p.183-198, 2012.

MALLARDI, Manuel; FERNÁNDEZ, Emiliano; MUSSO, María. Organismos internacionales de crédito y políticas sociales en América Latina. Plaza Pública, Número especial, p.64-74, 2015. Recuperado de (19/8/20):

https://revistaplazapublica.files.wordpress.com/2016/03/ne-9.pdf

MARANGOS, John. The evolution of the anti-Washington Consensus debate: From 'Post -Washington Consensus' to 'After the Washington Consensus'. Competition \& Change, v.12, n.3, p.227-244, 2008.

MAUL, Daniel. La Organización Internacional del Trabajo: 100 años de políticas sociales a escala mundial. Ginebra: OIT, 2019.

MERLING, Lara. IMF framework on social spending out of step with international standards. Bretton Woods Project, 2019. Recuperado de (19/8/20): https://www.brettonwoodsproject.org/2019/07/imf-framework-on-social-spending-out-of-step-withinternational-standards/.

MORANDE L., Felipe. A casi cuatro décadas del Consenso de Washington ¿Cuál es su legado en América Latina?. Estudios Internacionales, n.185, 2016.

NEMIÑA, Pablo; LARRALDE, Juan. Prestamista, garante y deudor: El FMI en América Latina y el Caribe en la década posterior a la crisis financiera. Sociedade e Cultura, n.23, 2020.

OIT. La Junta de Jefes Ejecutivos del Sistema de las Naciones Unidas pone la creación de empleos y la protección social en el centro de la atención de las iniciativas conjuntas contra la crisis. 2010. Disponível em: https://www.ilo.org/jobspact/news/WCMS_123986/lang--es/index.htm. Acesso em: 19 ago. 2020.

OIT. La estrategia de Desarrollo de los Sistemas de Seguridad Social de la OIT. El papel de los Pisos de Protección Social en América Latina y el Caribe. Lima: OIT, 2014.

OIT. Piso de Protección Social para una globalización equitativa e inclusiva. Ginebra: OIT. 2011.

OIT. Por una globalización justa El papel de la OIT. Ginebra: OIT, 2004.

ÖNIŞ, Ziya; ŞENSES, Fikret. Rethinking the Emerging Post-Washington Consensus. Development and Change, v.36, n.2, p.263-290, 2005.

PARK, Susan; VETTERLEIN, Antje (eds). Owning development: creating policy norms in the IMF and the World Bank. Cambridge: Cambridge University Press, 2010.

RIGGIROZZI, Pía; TUSSIE, Diana. Rethinking our region in a post-hegemonic moment. Post-hegemonic regionalism in the Americas. Towards a Pacific vs. Atlantic divide. En BRICEÑO-RUIZ J., MORALES, I. (Ed). Post-Hegemonic Regionalism in the Americas. Toward a Pacific-Atlantic Divide?, p.16-31. London: Routledge, 2017.

RIGGIROZZI, Pía. Social Policy, Inequalities and the Battle of Rights in Latin America. Development and Change, v.51, n.2, p.506-522, 2020.

SERRA, Narcís; SPIEGEL, Shari; STIGLITZ, Joseph E. Introduction: From the Washington Consensus Towards a New Global Governance. In SERRA, N.; SPIEGEL, S.; STIGLITZ, J. E. (Eds.). The Washington Consensus Reconsidered, p.3-13, Oxford: Oxford University Press, 2008.

STANDING, Guy. Precariado. Una carta de derechos. Madrid: Capitán Swing, 2014.

STIGLITZ, Joseph. More instruments and broader goals: Moving toward the post-Washington Consensus. WIDER Annual Lecture, Helsinki, 7 January, 1998. Recuperado de (19/8/20):

https://www.globalpolicy.org/component/content/article/209/43245.html

TOWNSEND, Peter. From Universalism to Safety Nets: The Rise and Fall of Kenynesian Influence on Social Development. En MKANDAWIRE, Thandika (ed.), Social Policy in a Development Context. London: Palgrave, p.37-62, 2004.

VALENCIA LOMELÍ, Enrique. Las transferencias monetarias condicionadas como política social en América Latina. Un balance: aportes, límites y debates. Annual Review of Sociology, v.34, p.499-524, 2008.

VETTERLEIN, Antje. Economic Growth, Poverty Reduction and the Role of Social Policies: The Evolution of the World Bank's Social Development Approach. Global Governance, v.13, n.4, p.513-533, 2007.

VETTERLEIN, Antje. Understanding Policy Change as Position-Taking: The IMF and Social Policies in Times of Crisis. In MCBRIDE, S.; MAHON, R.; BOYCHUK, G.W. (Eds). After '08: Social Policy and the Global Financial Crisis, p.87-104, Vancouver: UBC Press, 2015. 
WILLIAMS, David. Ownership, sovereignty and global governance. Econstor. University of Oxford, Global Economic Governance Programme, 2006.

WOODS, Ngaire. The globalizers: the IMF, the World Bank, and their borrowers. Ithaca: Cornell University Press, 2007.

ZURBRIGGEN, Cristina. Gobernanza: una mirada desde América Latina. Perfiles Latinoamericanos, v.19, n.38, p.39-64, 2011.

Funciones de colaboración ejercidas

Pablo Nemiña

Julián Echandi

Concepción: Metodología: Validación; Curación de datos; Gestión del proyecto; Visualización; Recursos; Adquisición de financiación; Investigación; Supervisión; Redacción (borrador original); Redacción (revisión y edición);

Concepción; Validación; Visualización; Análisis formal; Investigación; Redacción(borrador original);

Información proporcionada por los(as) autores(as) según la Taxonomía de Funciones de Colaboración (CRediT) 